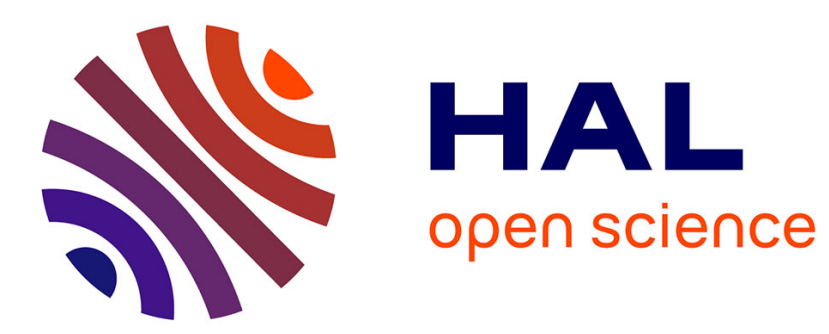

\title{
Aeroelastic effects on wave propagation and sound transmission of plates and shells
}

F Errico, F Franco, S de Rosa, G. Petrone, Mohamed Ichchou

\section{To cite this version:}

F Errico, F Franco, S de Rosa, G. Petrone, Mohamed Ichchou. Aeroelastic effects on wave propagation and sound transmission of plates and shells. AIAA Journal, 2019, 10.2514/1.J058722 . hal-02394021

\section{HAL Id: hal-02394021 \\ https://hal.science/hal-02394021}

Submitted on 4 Dec 2019

HAL is a multi-disciplinary open access archive for the deposit and dissemination of scientific research documents, whether they are published or not. The documents may come from teaching and research institutions in France or abroad, or from public or private research centers.
L'archive ouverte pluridisciplinaire HAL, est destinée au dépôt et à la diffusion de documents scientifiques de niveau recherche, publiés ou non, émanant des établissements d'enseignement et de recherche français ou étrangers, des laboratoires publics ou privés. 


\title{
Aeroelastic effects on wave propagation and sound transmission of plates and shells
}

\author{
F. Errico * \\ Ecole Centrale de Lyon, Écully, France, 69134. \\ Universitá degli Studi di Napoli Federico II, Napoli, Italy, 80125. \\ F. Franco ${ }^{\dagger}$, S. De Rosa ${ }^{\ddagger}$ and G. Petrone $\S$ \\ Universitá degli Studi di Napoli Federico II, Napoli, Italy, 80125. \\ M. Ichchou ${ }^{\text {II }}$ \\ Ecole Centrale de Lyon, Écully, France, 69134.
}

Modal approaches are often preferred to the wave-based ones for the evaluation of instability conditions in classical non-lifting aeroelasticity of plates and shells. Here, within a wave-based finite element framework, sub- and super-sonic aerodynamic models are introduced to analyse the effect of self-excited aerodynamic loading terms on the dispersive characteristics of structural waves. The method is validated by using a specific literature test-case and is applicable both on isotropic and multi-layered flat and curved structures. The sound transmission is also computed under sub- and super-sonic turbulent boundary layer excitations: the effect of including or neglecting the aeroelastic coupling is discussed.

\section{Nomenclature}

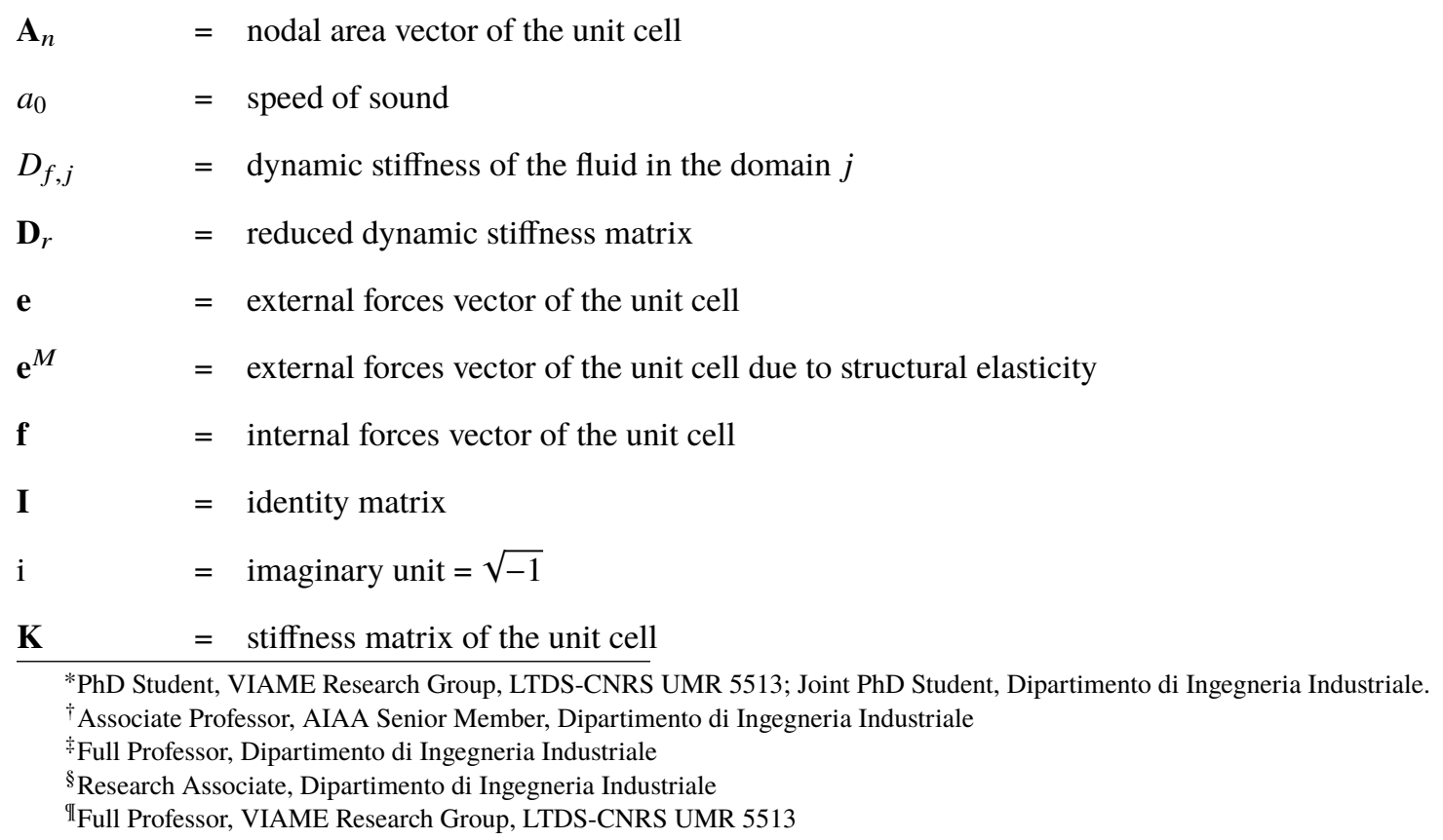




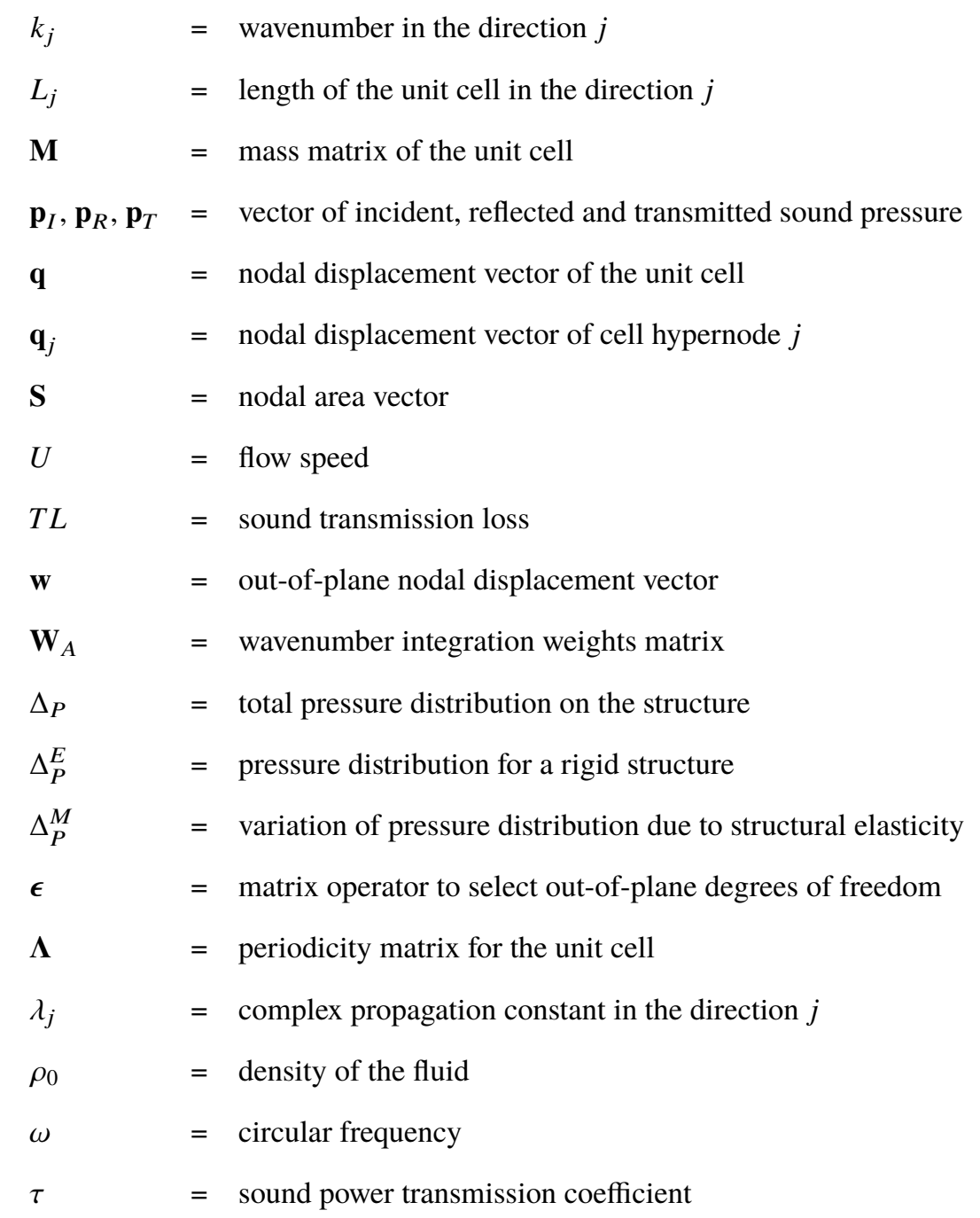

\section{Introduction}

$\mathrm{T}$

HE aeroelasticity of plates and shells, extensively studied in the last decades, faces the difficulty of distinguishing between the self-induced vibration components and the external one. Phenomenological non-linearities induce fatigue failures instead of catastrophic instantaneous failures, typical of the aeroelasticity of lifting surfaces [1, 2]. In this context, the modal approach is often preferred to a wave-based one, because it allows a clear evaluation of the flutter conditions, analysing the effect of the aerodynamic-induced forces on each structural mode [1-6]. Consequently, the effects of aerodynamics on the elastic structural waves' propagation, are rarely studied and few works are present in the literature [4, 6, 8$]$.

The infinite-plate problem, has been developed by Miles [4] and Crighton \& Oswell [9]. The first (Ref. [4]) presents a work discussing the flutter of an isotropic infinite panel in a two-dimensional incompressible flow, identifying the flutter conditions versus the circular frequency in terms of wave speed. The latter (Ref. [9]) develops an analytical 
model that describes, in a neutral stability zone, some highly unusual wave propagation effects in presence of flow.

On the other hand, the effect of mean flow on cylindrical structures has been then studied by the same Miles [5] and others authors [10, 11]. In particular, Peake (Ref. [11]) provides a closed-form dispersion relation for circumferential waves in infinite cylinders in presence of incompressible external flow; this is here used for validation purposes. Regarding the flow-induced vibrations or sound transmission in presence of a turbulent boundary layer excitation, the classic approaches are limited to the blocked pressure assumption [12]. The flow back reaction is taken into account rarely in the literature [1, 13]. In addition, the computational cost issues related to mesh requirements due to the lower values of the convective wavelengths in most of the practical cases, remain one of the main limits of standard approaches. Recently, through the use of wave-based finite element approaches, a strong reduction of computational cost was demonstrated for finite flat and curved structures under turbulent boundary layer excitation [14]. Hereby, both subsonic and supersonic aerodynamic flows are introduced in models of homogenised periodic plates and shells; the elastic wave propagation is thus investigated, assuming that only one side of the structural domain is wetted by the flow. The sound transmission loss is also computed, using a set of aleatory surface waves (Ref. [15]) and discussed versus aeroelastic effects.

The novelties targeted in the present work are mainly based on including mean-flow effects in recent Wave-based Finite Element schemes for the simulation of sound transmission. In fact, since blocked pressure assumptions (one-way coupling) are typically used in most classic simulations of flow-induced vibrations and sound transmission in aeroacousto-elastic problems, by including the flow effects on the structural dispersion, wave-based approaches can partially release this weak coupling condition.

\section{The Numerical Approach}

The method here presented is based on the Wave Finite Element Method (WFEM) [16, 17].

Within a periodic or homogeneous structural framework, the finite element modelling of a single elementary cell is required (see Fig. 1). The mass and stiffness matrices of the cell, respectively $\mathbf{M}$ and $\mathbf{K}$, are extracted. One can use analytical formulations for the finite elements and get the mass and stiffness matrices by his own by applying Hamilton's principle or using handbook for FE formulations. Otherwise, any commercial finite element software can be used, with all the included libraries; in this work, the software ANSYS is used and the elements employed to model the unit-cell FE models are SOLID45 or SHELL181. The Bloch theorem, see Refs. [16], can be applied imposing a link among the nodal degrees of freedom of the cell, as in Eq. (11). Assuming an homogeneous-in-plane structure, the nodal displacement vector of the cell, $\mathbf{q}$, can be thus expressed as a function of the ones of a single corner, $\mathbf{q}_{1}$, which represent the vector of degrees of freedom of the hypernode 1 :

$$
\mathbf{q}=\Lambda \mathbf{q}_{1} ; \quad \mathbf{\Lambda}=\left[\mathbf{I} ; \lambda_{x} \mathbf{I} ; \lambda_{y} \mathbf{I} ; \lambda_{x} \lambda_{y} \mathbf{I}\right]
$$




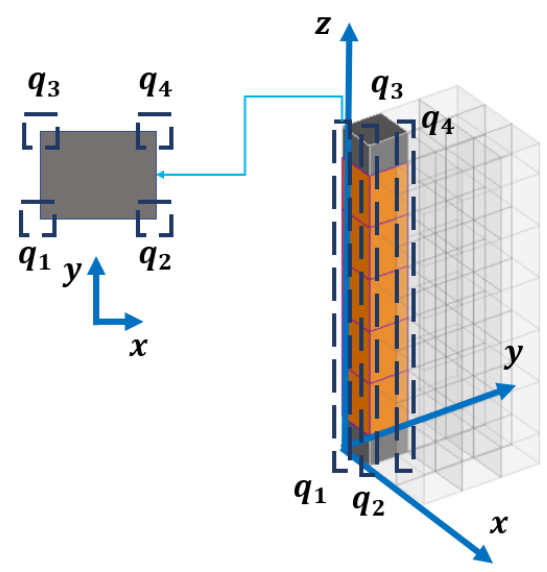

Fig. 1 An example of FE model of a periodic cell and the ordering of the hypernodes on the corners.

where $\lambda_{x}$ and $\lambda_{y}$ are the complex propagation constants, which can be written as:

$$
\lambda_{x}=e^{-\mathrm{i} k_{x} L_{x}} ; \quad \lambda_{y}=e^{-\mathrm{i} k_{y} L_{y}}
$$

where $L_{x}$ and $L_{y}$ are the sizes of the cell in the plane X-Y, while $k_{x}$ and $k_{y}$ are the wavenumbers (for each wave type) in the $\mathrm{X}$ and $\mathrm{Y}$ direction, respectively; $\mathbf{I}$ is the identity matrix. Identical conditions are applicable also to the force vectors, f (internal) and $\mathbf{e}$ (external). By exploiting the periodic link and multiplying the dynamic stiffness equation by the Hermitian of the periodicity matrix, $\boldsymbol{\Lambda}$, the reduced dynamic stiffness equation can be derived:

$$
\boldsymbol{\Lambda}^{H}\left[\mathbf{K}-\omega^{2} \mathbf{M}\right] \mathbf{\Lambda} \mathbf{q}_{1}=\boldsymbol{\Lambda}^{H} \boldsymbol{\Lambda} \mathbf{f}+\boldsymbol{\Lambda}^{H} \boldsymbol{\Lambda} \mathbf{e}
$$

where $\mathbf{f}$ and $\mathbf{e}$ are the nodal vectors of internal and external forces respectively; $\omega$ the circular frequency. Because of the equilibrium of internal forces between consecutive cells, the term $\boldsymbol{\Lambda}^{H} \boldsymbol{\Lambda} \mathbf{f}$ in Eq. (3) is null.

At this stage, when no external force is applied, the problem in Eq. (3) is representative of a three-parametric eigenproblem in $\lambda_{x}, \lambda_{y}$ and $\omega$, that can be solved by imposing two variables at the time [17]. By solving the quadratic eigenvalue problem in $\lambda_{x}$ or $\lambda_{y}$, the dispersion curves of the structure can be derived, without flow effects.

This periodic conditions can be also imposed along circular (periodic) paths, in order to study wave propagation in shells. While the mass and stiffnes matrices of the cell can still be extracted from flat FE cell models, the curvature is simulated pre and post multiplying them with rotational matrices [15, 17]. The propagation constant $\lambda_{x}$, will be, in this case, representative of the circumferential wave propagation constant and the solutions of the eigenvalue of Eq. (3) will be the circumferential wavemodes of the shell.

It is worth to remark how the periodic or homogeneous conditions in Eq. (3) are only employed to use a unit-cell 


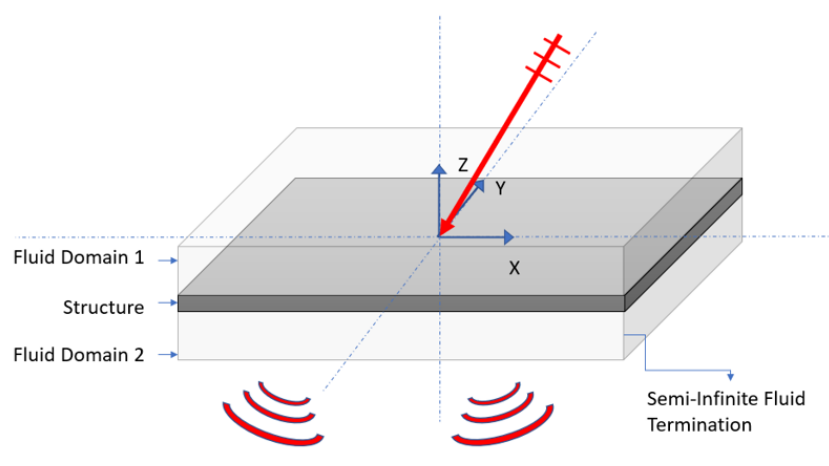

Fig. 2 Illustration of an infinite the structure excited by a plane wave and radiating sound.

modelling approach and do not target any study regarding periodic effects on aeroelastic response (see Refs. [18, 19]). On the other hand, the unit-cell approach proposed here, would fit perfectly with the study of periodic structures under aerodynamic load.

\section{A. Fluid-Structure Coupling}

When a forcing wave excites the structure, as in Fig. 2, with an amplitude $p_{I}$, the structure transmits and reflects structure-borne sound waves in the excited (subscript 1) and radiating (subscript 2) fluid domains. Therefore, the nodal forces acting on the unit-cell, can be expressed using the sound pressure amplitudes and the nodal surfaces, that can be calculated as follows (see Ref. [15]):

$$
\mathbf{e}_{1}=\mathbf{S} \cdot\left(\mathbf{p}_{I}+\mathbf{p}_{R}\right) \mathbf{w}_{1}-\mathbf{S} \cdot \mathbf{p}_{T} \mathbf{w}_{2}
$$

where $\mathbf{S}$ is vector of the nodal areas, $\mathbf{w}_{1}$ and $\mathbf{w}_{2}$ are vectors of all zeros and ones in the position corresponding to the out-of-plane displacements degrees of freedom of the nodes belonging to the surfaces in contact with the flow (effective for the sound transmission), $\mathbf{p}_{I}, \mathbf{p}_{R}$ and $\mathbf{p}_{T}$ are the amplitudes of the excited, reflected and transmitted pressure waves, respectively.

From the continuity of the normal particle velocity on the excited and radiating surfaces, the dynamic stiffnesses of the fluid are expressed as (see Ref. [15]):

$$
D_{f, 1}=\frac{-\mathrm{i} \rho_{1} \omega^{2}}{k_{Z, 1}} \quad D_{f, 2}=\frac{-\mathrm{i} \rho_{2} \omega^{2}}{k_{Z, 2}}
$$

where $\rho_{1}$ and $\rho_{2}$ are the fluid densities, $k_{Z, 1}$ and $k_{Z, 2}$ the wavenumber components in $Z$ (i.e. out-of-plane) and $D_{f, 1}$ and $D_{f, 2}$ the dynamic stiffness of the fluid in the incident and radiating domains, respectively.

Exploiting the relationships of Eqs. 4 and 5 in Eq. 3 the final dynamic stiffness equation becomes:

$$
\left[\mathbf{D}_{r}+\left(D_{f, 1} \mathbf{w}_{1}+D_{f, 2} \mathbf{w}_{2}\right) \mathbf{S} \boldsymbol{\Lambda}^{H} \boldsymbol{\Lambda}\right] \mathbf{q}_{1}=2 p_{I} \mathbf{w}_{1} \mathbf{S} \boldsymbol{\Lambda}^{H} \boldsymbol{\Lambda}
$$


The algebraic system in Eq. 6 6 can be solved in $\mathbf{q}_{1}$ and the sound power transmission coefficient $\tau$, associated with the couple of forcing wavenumbers $k_{X}$ and $k_{Y}$, is derived as follows:

$$
\tau\left(k_{X}, k_{Y}\right)=\frac{\left(k_{Z, 2} / \rho_{2}\right) \mathbf{S}\left|\left(D_{f, 2} \mathbf{w}_{2} \mathbf{q}_{1}\right)^{2}\right|}{\left(k_{Z, 1} / \rho_{1}\right) \mathbf{S}\left|\mathbf{p}_{I}^{2}\right|} .
$$

Finite size effects can be accounted using a baffled window approach [15, 20]. However, a real external load, corresponding, for example, to a turbulent boundary layer, can be simulated using a weighted integration of surface waves in the wavenumber space (see Ref. [15]). Finally, an integration of the transmission coefficient is performed, in the wavenumber domain, and the final transmission loss calculated as such:

$$
\operatorname{TL}(\omega)=-10 \log _{10}\left(\frac{\iint \tau\left(k_{X}, k_{Y}\right) \times W_{A}\left(k_{X}, k_{Y}, \omega\right) \mathrm{d} k_{X} \mathrm{~d} k_{Y}}{\iint W_{A}\left(k_{X}, k_{Y}, \omega\right) \mathrm{d} k_{X} \mathrm{~d} k_{Y}}\right)
$$

where $W_{A}\left(k_{X}, k_{Y}, \omega\right)$ is the normalized wave amplitude for the wavenumber couple involved in the integration process. The only requirements for this approach, as discussed in Ref. [15], are imposed by the homogeneity or periodicity of the structure, and by the knowledge of the wall pressure spectrum for the excitation model. Convergence studies are present in Ref. [15].

\section{Aerodynamic Loading in Periodic Framework}

To simulate a mean flow, working on one side of an infinite structural domain, a specific aerodynamic theory has to be used to model the self excited aerodynamic components of the load. Here, supersonic flows are described using the simplest aerodynamic theory, the Piston Theory [1, 21], while for subsonic aerodynamic flows, an approximated formula for incompressible flows is used, as proposed by Dowell [1].

It is worth to notice how, in the case of transonic flows (Mach $\approx 1$ ), where the aeroelastic effects are most important and flutter phenomena more critical, simple aerodynamic theories do not exist (see Refs. [22-24]), while numerical approaches predict the presence of shock waves (Ref. [25, 26]). Here, linear theories are used for the supersonic and subsonic flow regimes, but similar approaches are not possible for transonic flows since moving shock waves and partially subsonic flow-fields are inherently nonlinear [22]. Differences between experiments and linear theories in the transonic regime are also discussed in [23, 24].

The aerodynamic pressure can be normally considered as made up of two components (see Ref. [1]), as in Eq. (99: one is given by the pressure fluctuations for a rigid body $\left(\Delta_{P}^{E}\right)$; the second is dependent on the structural motion/elasticity $\left(\Delta_{P}^{M}\right)$. The following developments are connected to the second of these contributes.

$$
\Delta_{P}=\Delta_{P}^{M}+\Delta_{P}^{E}
$$


Finally, using the notation of Eq. 97, the dynamic stiffness equation of a periodic/homogenised unit-cell can be written as in Eq. [10].

$$
\boldsymbol{\Lambda}^{H}\left[\mathbf{K}-\omega^{2} \mathbf{M}\right] \boldsymbol{\Lambda} \mathbf{q}_{1}=\boldsymbol{\Lambda}^{H} \boldsymbol{\Lambda} \mathbf{f}+\boldsymbol{\Lambda}^{H} \boldsymbol{\Lambda}\left(\mathbf{e}^{M}+\mathbf{e}^{E}\right)
$$

As discussed by Dowell in Ref. [1], this assumption of linear superposition of effects, implies that the plate motion and the consequent portion of aerodynamic pressure are small enough to not modify the turbulent pressure fluctuations outside.

\section{A. The Piston Theory}

The Piston Theory, valid from Mach > 1.5, assumes that the pressure fluctuations in any point of the system are linearly independent, by neglecting the effects of spatial and temporal memory (see Refs. [1, 21]). Using the notation of Eq. (3), the self-excited force terms can be written as a function of the convective and continuity derivative, [1, 3, 4, 21]:

$$
\mathbf{e}^{M}=-\rho_{0} a_{0} \mathbf{A}_{n}\left(\frac{\partial \mathbf{w}}{\partial t}+U \frac{\partial \mathbf{w}}{\partial x}\right)
$$

where $\mathbf{w}$ represents the vector of the out-of-plane displacements of the structural nodes belonging to the surfaces in contact with the flow (coordinate $\mathrm{Z}$ in Fig. 11, $\rho_{0}$ is the fluid density, $a_{0}$ the sound speed, $\mathbf{A}_{n}$ the nodal area vector, [27], and $U$ the flow-speed.

The out-of-plane displacements can be expressed by multiplying $\mathbf{q}$ for a matrix $(\boldsymbol{\epsilon})$ of 0 and 1 in the positions corresponding to the target degrees of freedom (i.e. the translations in Z). For example, the matrix $\epsilon$, can be built as such:

$$
\epsilon_{i, j}= \begin{cases}1 & \text { if } \quad j=3 ; i=1, N \\ 0 & \text { else }\end{cases}
$$

where $i$ represents the number of nodes, and $j$ the nodal degree of freedom (i.e. 3 corresponds to translation along Z).

In a periodic structural framework, the spatial derivative in Eq. 11] is a function of the structural propagation constant ( $\lambda_{x}$, assuming $\mathrm{X}$ as the flow direction), and can be expressed, using a simple numerical scheme for the first derivative, as follows:

$$
\frac{\partial \mathbf{w}}{\partial x}=\boldsymbol{\Lambda}\left(\frac{\lambda_{x}-1}{L_{x}}\right) \boldsymbol{\epsilon} \mathbf{q}_{1}
$$

The final dynamic stiffness equation can be derived, as in Eq. (14), substituting Eqs. (11) and (13) in Eq. (3).

$$
\boldsymbol{\Lambda}^{H}\left[\mathbf{K}-\omega^{2} \mathbf{M}-\mathrm{i} \omega \rho_{0} a_{0} \mathbf{A}_{n} \boldsymbol{\epsilon}+U \rho_{0} a_{0} \mathbf{A}_{n} \boldsymbol{\epsilon}\left(\frac{\lambda_{x}-1}{L_{x}}\right)\right] \boldsymbol{\Lambda} \mathbf{q}_{1}=\boldsymbol{\Lambda}^{H} \boldsymbol{\Lambda} \mathbf{f}=0
$$

The assumption in Eq. (13) is fundamental and is possible due to the periodic links between wavefields in the structure 
(Eq. (3)). The convective derivative becomes periodic in Eq. (13) since the displacements field is periodic. However, this assumption, while true for an isolated structure, is an approximation in the case of a loaded structure. Nevertheless, the mean-flow effects, are here hidden in additional damping and stiffness terms in the new dynamic stiffness equation (Eq. (14)). The additional damping is proportional to the circular frequency, but, as discussed in Ref. [1], it is not a dominant term. A strong variation of the results of Eq. (3) versus the Eq. (14) ones, is given by the additional stiffness terms, which are proportional to the stream-wise elastic waves' propagation coefficient.

\section{B. Subsonic Aerodynamic Flows}

Similarly, when a different aerodynamic model is investigated, as a subsonic incompressible flow, the spatial derivatives can still be expressed as a function of the structural waves' propagation constants, using, generally, numerical schemes of higher order. The aerodynamic forces connected to the structure motion can be expressed, for low Mach number subsonic flows (incompressible flows), using again linear models, that do not consider spatial and temporal memory, as follows (see Ref. [1]):

$$
\mathbf{e}^{M}=\frac{\rho_{0} U^{2} \mathbf{A}_{n}}{\pi}\left[\frac{\partial^{2} \mathbf{w}}{\partial x^{2}}+\frac{2}{U} \frac{\partial^{2} \mathbf{w}}{\partial x \partial t}+\frac{1}{U^{2}} \frac{\partial^{2} \mathbf{w}}{\partial t^{2}}\right]
$$

Substituting Eq. (15) in Eq. (3), using a second order numerical scheme for the second order spatial derivatives, the final dynamic stiffness matrix is obtained:

$$
\boldsymbol{\Lambda}^{H}\left[\left(\mathbf{K}+\frac{2 \rho_{0} U^{2} \mathbf{A}_{n p}}{L_{x}^{2}} \boldsymbol{\epsilon}\right)-\omega^{2}\left(\mathbf{M}-2 \rho_{0} \mathbf{A}_{n p} \boldsymbol{\epsilon}\right)+\frac{\rho_{0} U \mathbf{A}_{n p}}{L_{x}}\left(2 \mathrm{i} \omega+\left(2 \mathrm{i} \omega-\frac{U}{L_{x}}\right) \lambda_{x}-\frac{U}{L_{x}} \lambda_{x}^{-1}\right) \boldsymbol{\epsilon}\right] \boldsymbol{\Lambda} \mathbf{q}_{1}=0
$$

where $\mathbf{A}_{n p}=\frac{A_{n}}{\pi}$.

Here, the derivation schemes used are:

$$
\begin{gathered}
\frac{\partial^{2} \mathbf{w}}{\partial x^{2}}=\boldsymbol{\Lambda}\left(\frac{\lambda_{x}-2+\lambda_{x}^{-1}}{L_{x}^{2}}\right) \boldsymbol{\epsilon} \mathbf{q}_{1} \\
\frac{\partial^{2} \mathbf{w}}{\partial x \partial t}=-i \omega \boldsymbol{\Lambda}\left(\frac{\lambda_{x}-1}{L_{x}}\right) \boldsymbol{\epsilon} \mathbf{q}_{1} \\
\frac{\partial^{2} \mathbf{w}}{\partial t^{2}}=-\omega^{2} \boldsymbol{\Lambda} \boldsymbol{\epsilon} \mathbf{q}_{1}
\end{gathered}
$$

Comparing Eq. (3) and Eq. (16), additional stiffness, damping and inertia terms, dependent on the flow speed and density, can be observed. In addition, some explicit influence on the structural waves' propagation appears with stream-wise (terms proportional to $\lambda_{x}$ ) and cross-wise (terms proportional to $\lambda_{x}^{-1}$ ) aerodynamic components. Again, the eigenvalue problem is parametric and can be solved by fixing two parameters between $k, \omega$ and $U$. 
It is interesting to observe the absence of non-linearities in the eigenvalue problem.

\section{Numerical Results}

Here, the eigenvalue problems of Eqs. (3), (14) and (16) are solved, for specific test-cases. A validation for flat plates in supersonic flows is performed using the travelling wave approach by Miles (see Refs. [4-6]). The dispersion curves for shells are also computed and a validation with the Donnel-Mushtari theory with and without flow is performed (see Ref. [11]). In both cases the external flow is assumed to be inviscid air.

Finally, the sound transmission under an external turbulent boundary layer excitation, is computed and compared when aeroelastic effects are accounted in the model, for both supersonic and subsonic flows. The approach used for fluid-structure coupling and random load simulation, within a wave finite element framework, follows the same proposed in [15]. Despite the numerical complexity in Eqs. [14] and [16, the proposed numerical framework allows simpler evaluations of the effective sound transmission loss, due to a turbulent boundary layer load, than classic modal approaches [13, 28, 29].

\section{A. Wave Propagation in Plates and Shells}

First, a 2mm-thick flat aluminium plate is considered. The test-case analysed by Miles, (see Ref. [4]), is reproduced and the analytic solution is used to validate the proposed approach. In Fig. 3 the bending wave speed and wavenumbers of the plate in the stream-wise direction, are plotted and compared to the case a one-sided flow (Mach 1.6) is simulated; the present approach is compared to the approach of Miles [4]. A good agreement with the reference solution is observed, as a variation of the bending wavenumbers with respect to the purely structural case, in the lowest frequency bands. The increase of the wavenumbers is somewhat representing a reduction of bending stiffness of the plate, caused by the action of the flow, that vanishes when the frequency increases, approaching the purely structural solution.

Differently, in Fig. 4 the dispersion curves of an aluminium shell (2mm-thick; $1.5 \mathrm{~m}$ radius), with and without flow, are plotted and compared, both in the axial direction (stream-wise), and the circumferential one. The use of the aerodynamic model presented in Section [II] (Piston Theory), which excludes three-dimensional effects is presented in some works in the literature [30, 31]. In fact, the mitigation of circumferential cross-flow pressure gradients by means of flow viscosity, justifies the use of the linear piston theory as a simplified aerodynamic model for shells [32].

In Fig. 4 a, the wavenumbers in the direction of the flow (axial direction) are increased with respect to the purely structural case (absence of flow), as in the case of a flat plate (see Ref. [4]). On the other hand, for circumferential waves (Fig. 4p), the effects mainly visible around and above the ring frequency, because of the higher stiffness of shells before the first extensional mode. In both cases, for increasing frequency, the dispersion curves converge to the ones of the purely structural case (absence of flow) and only bending waves are affected by the presence of the flow; shear and longitudinal wavemodes are not excited by the pressure fluctuations. A good agreement with the reference solutions 

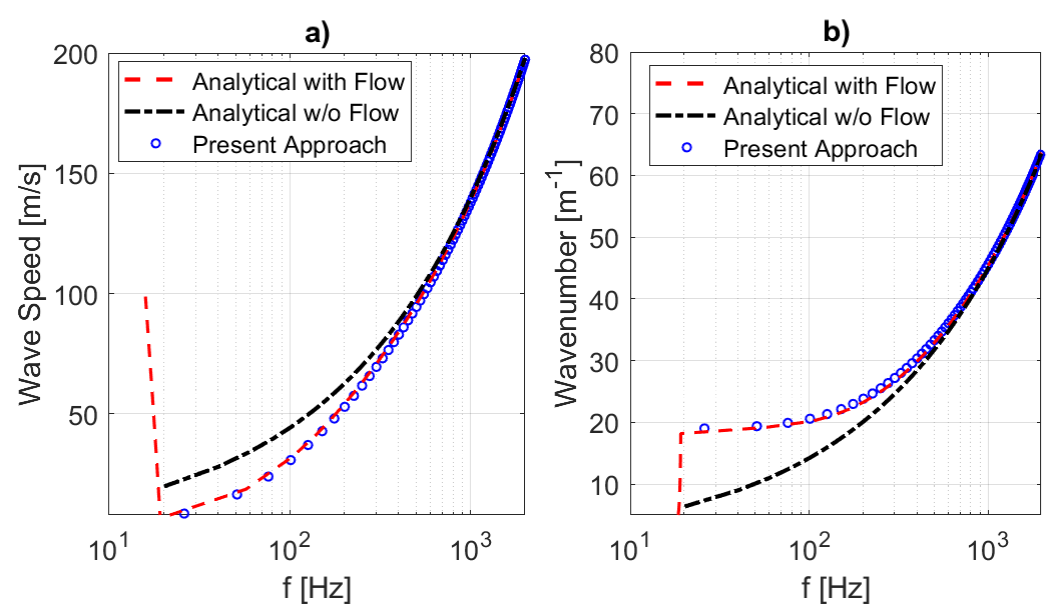

Fig. 3 Stream-wise bending waves in a $2 \mathrm{~mm}$-thick flat aluminium panel with a one-sided flow at Mach 1.6. Reference solution from [4, 6]: a) Wave Speed; b) Wavenumber.
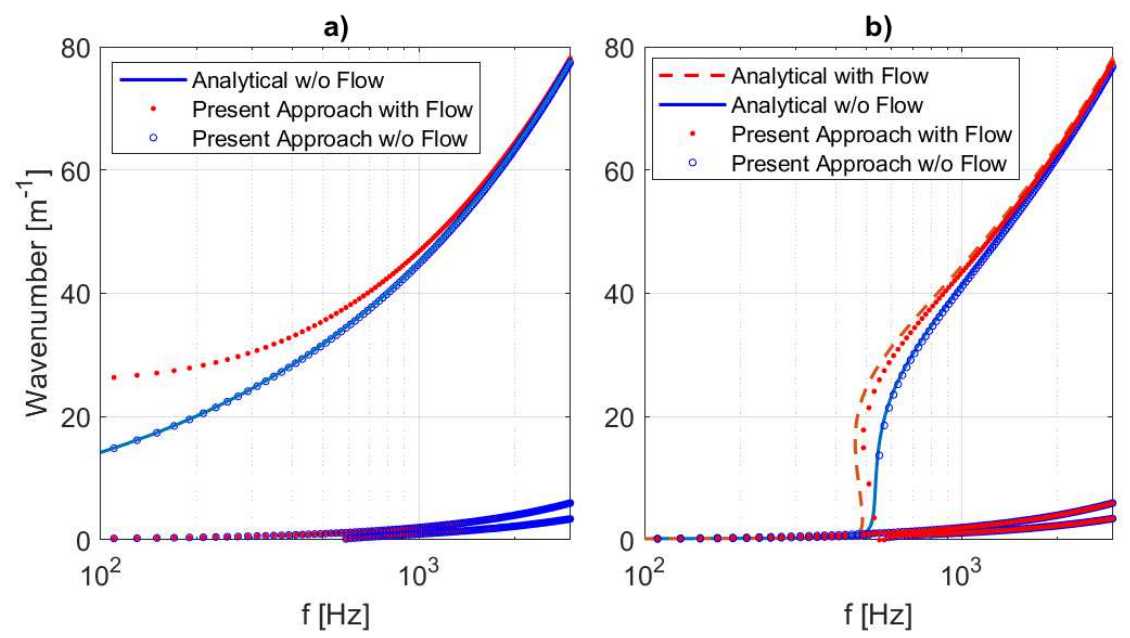

Fig. 4 Dispersion curves for an aluminium shell ( $2 \mathrm{~mm}$-thick, $1.5 \mathrm{~m}$ curvature) with a one-sided flow at Mach 2.5: a) Axial Waves; b) Circumferential Waves. Analytical model: Ref. [11].)

(see Refs. [11, 33]) is observed, with and without flow.

The effects of increasing flow speed and circumferential order are shown in Fig. 5 . In Fig. 5, a fixed circumferential order is considered and the flow speed is increased from Mach 2.5 to 4.5; a larger wavenumber region is affected, for increasing speed, inducing a distorted transition to the flat-plate bending behaviour after the cut-on frequency. Differently, in Fig. 6 the effect of increasing the circumferential order from 1 to 10 is shown; the effects seem independent on the presence of the flow, being coherent with the variation one has for the purely structural case (see Ref. [34]). A further validation is also provided in Figs. 5 and 6 by the presence of the analytical curves derived from [11]. 


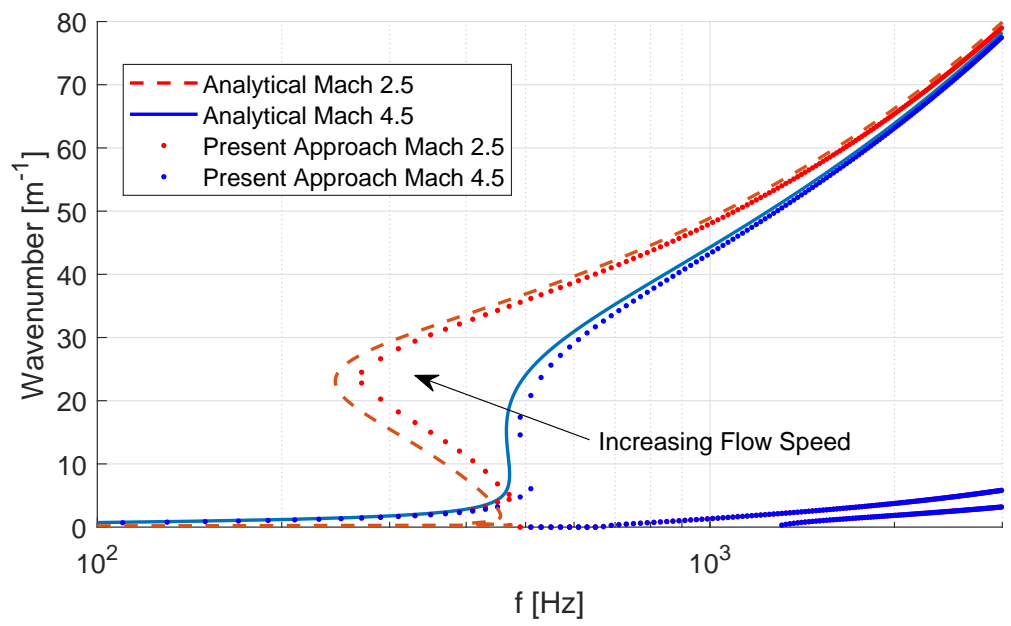

Fig. 5 Dispersion curves for an aluminium shell ( $2 \mathrm{~mm}$-thick, $1.5 \mathrm{~m}$ curvature) with a one-sided flow at Mach 2.5 and 4.5. Analytical model: Ref. [11].

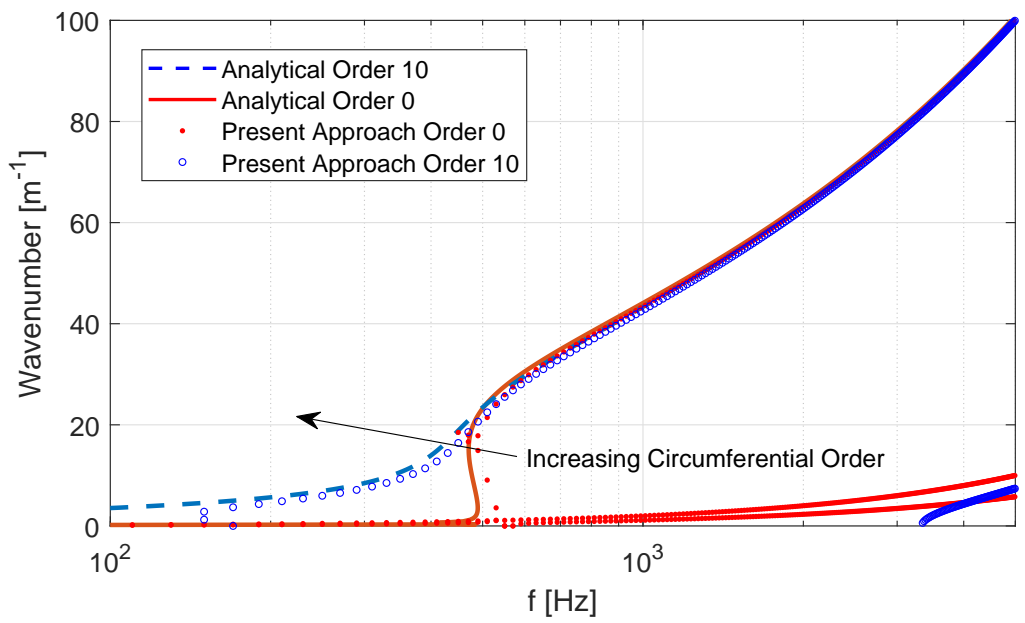

Fig. 6 Dispersion curves for an aluminium shell ( $2 \mathrm{~mm}$-thick, $1.5 \mathrm{~m}$ curvature) with a one-sided flow at Mach 2.5, for increasing circumferential order. Analytical model: Ref. [11]. 

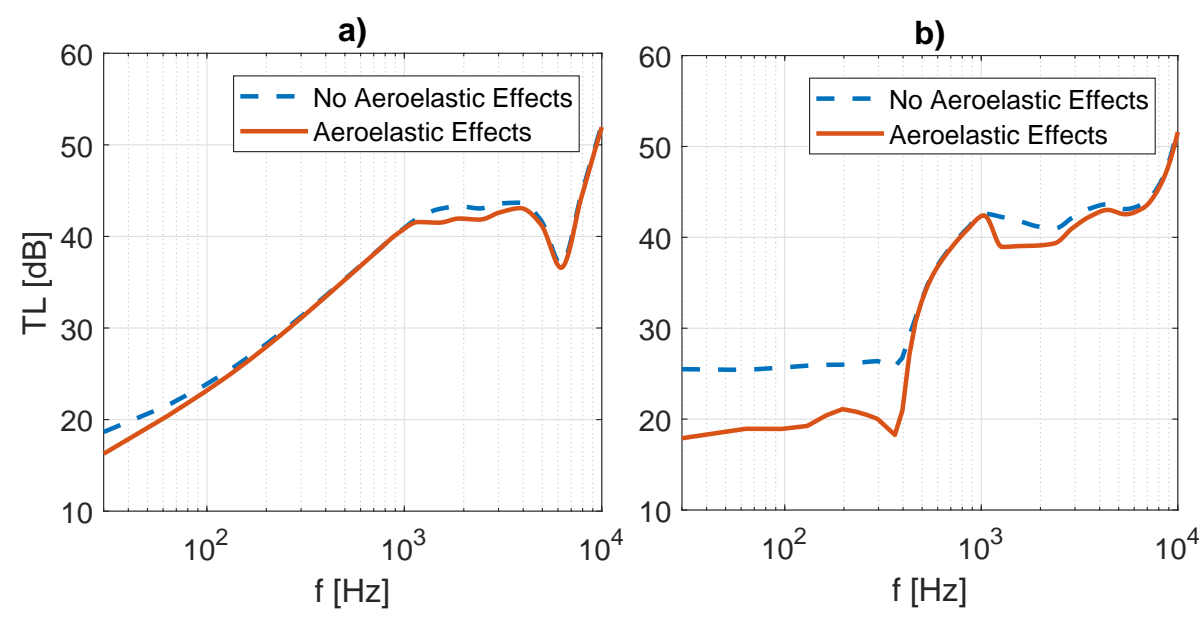

Fig. 7 Sound transmission under a supersonic turbulent boundary layer load (Mach 1.35): a) Flat Panel $\left(0.5 \times 0.3 \mathrm{~m}^{2}\right)$; b) Curved Panel $\left(0.5 \times 0.3 \mathrm{~m}^{2} ; 2 \mathrm{~m}\right.$ curvature $)$

\section{B. Sound Transmission under Turbulent Boundary Layer}

The effect of the mean flow on the sound transmission of flat and curved panels is here investigated. First, a supersonic flow is simulated (Mach 1.35) and a Cockburn-Robertson TBL model is used (see Ref. [35]) on flat and curved panels. In this case, the Piston Theory is used for the load contribution auto-generated by the structure elasticity $\left(\Delta_{P}^{M}\right.$ in Eq. 14p).

In Fig. 7. the sound transmission loss is affected by the aeroelastic effects in the low frequencies. This is caused by the strong alterations in bending waves' propagation, caused by the flow itself. However, differences are observed also before the acoustic coincidence $(\approx 6 \mathrm{kHz})$. In fact, the variation of wavemodes induced by the aeroelastic effects, eigenvectors from Eqs. (14), induces different structural reaction with respect to the case in absence of flow. The drop caused by the critical aerodynamic frequency is hardly visible being close to the acoustic coincidence $(\approx 6.7 \mathrm{kHz})$.

For the curved panel (Fig. 7b), before the ring frequency, the effects are strongly amplified.

On the other hand, a subsonic flow (Mach 0.5) is simulated in Fig. 8, using the TBL model of Corcos (see Ref. [36]). In this case, Eqs. (15) is used for the subsonic load contribution generated by the structural motion. Again, flat and curved panels are studied. Differently to the supersonic case, the effects of the flow are somewhat distributed in the whole frequency band. The aeroelastic effects induce a slightly higher transmission loss and a shift of the aerodynamic and acoustic coincidence to higher frequencies. It is a classic mass addition effect, that, in term of elastic waves, can be read as a monotonic increase of bending waves' wavenumbers versus frequency.

It must be underlined that the physical mechanism that induces an increase of the wavenumbers (or reduction of bending wave speed) in the structure, is different between the supersonic and subsonic aerodynamics. While in the first case, a reduction of dynamic stability plays a role (travelling flutter; see Ref. [4]), in the subsonic case is physically similar, as said, to an added mass effect. In the latter case, given the aerodynamic model applied here, the effects of the 

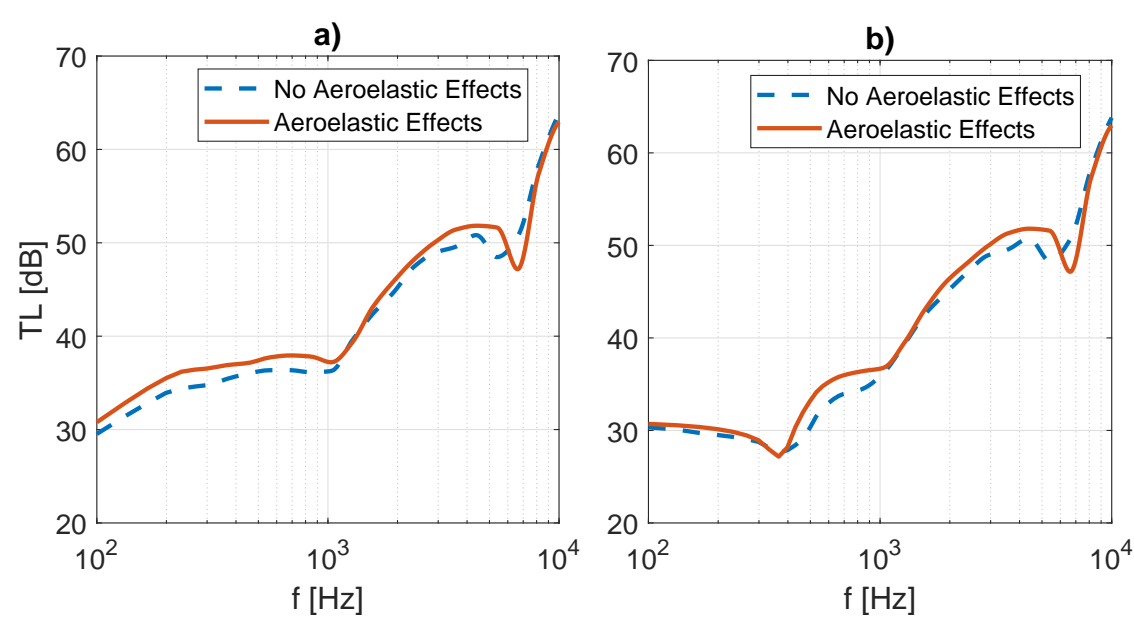

Fig. 8 Sound transmission under a subsonic turbulent boundary layer load (Mach 0.5): a) Flat Panel (0.7x0.5 $\left.m^{2}\right)$; b) Curved Panel $\left(0.7 \times 0.5 \mathrm{~m}^{2} ; 2 \mathrm{~m}\right.$ curvature).

Table 1 Materials' properties of the sandwich plate.

\begin{tabular}{ccc} 
& Skins & Core \\
\hline$E_{1}(\mathrm{GPa})$ & 69.0 & 0.3 \\
$v_{1,2}$ & 0.33 & 0.2 \\
$\rho\left(\mathrm{Kg} / \mathrm{m}^{3}\right)$ & 2742 & 48 \\
Thickness $(\mathrm{mm})$ & 1 & 10 \\
\hline
\end{tabular}

presence of the mean flow might be neglected.

A more complex example is illustrated in Fig. 9 with a sandwich panel under a subsonic turbulent boundary layer; the material data are in Table1. The mean-flow effects are observable in the whole frequency range and differently from the simple aluminium panel, the effects at higher frequencies seem to reduce the sound transmission loss. As expected (see Ref. [15]) the accuracy of the approach in the lowest frequency ranges is reduced. The present approach, in fact, considers a wave propagation to the infinite and does not take into account the boundaries of the finite structure (see Ref. [15]). One-dimensional wave-based finite element approaches (see Ref. [14]), on the contrary, can account for boundaries but require a higher computational cost. A comparison with a classic FEM approach (Ref. [14]) is also proposed in terms of computational cost in Table 2

Table 2 Computational cost comparison for an Intel(R) Core(TM) i7-7700 CPU 3.60GHz processor (16Gb RAM).

\begin{tabular}{ccccc}
\hline Method & Model & DoFs & Time/freq [sec] & Reduction Ratio \\
\hline FEM (Ref. [14]) & Full & $120 \times 10^{3}$ & 440 & - \\
Present Approach & Unit-cell & 888 & 35 & 12.6
\end{tabular}




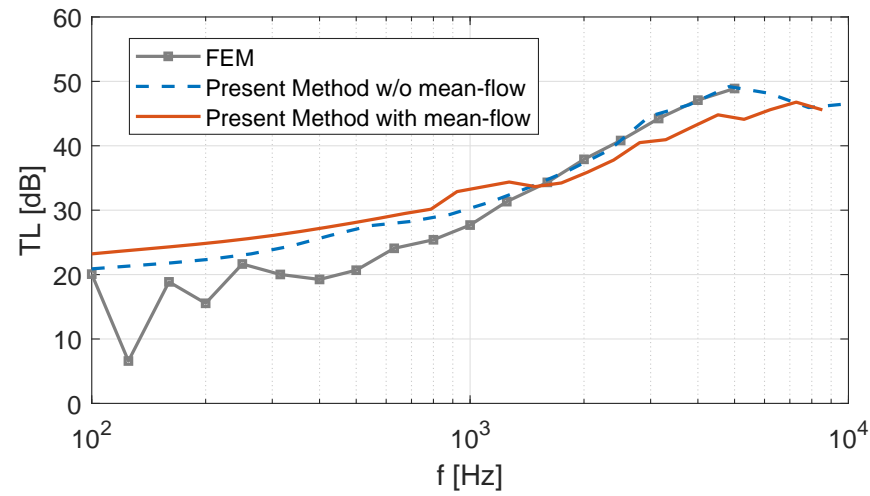

Fig. 9 Sound transmission loss of a sandwich flat panel $\left(0.8 \times 0.6 \mathrm{~m}^{2}\right)$ under a subsonic turbulent boundary layer load at Mach 0.62.

\section{Conclusion}

A procedure to account aeroelastic effects in the computation of dispersion curves and sound transmission of panels and shells is developed. The structural framework is based on the wave finite element method. The modelling of a single elementary cell is needed; homogeneous and multi-layered plates and shells can be studied with a reduced computational effort.

Once an aerodynamic theory is used, the components of the load connected to the pressure fluctuations that are produced by the motion of the flexible body, are described developing temporal and spatial derivatives in a periodic framework; the convective terms become function of the elastic waves' propagation constants and are injected in the new dynamic stiffness equation of the system.

A validation of the method is proposed using literature results for the travelling flutter of infinite thin plates (see Ref. [4]). The supersonic flow effects on the bending waves of a plate and shell are studied. The resulting sound transmission, under turbulent boundary layer excitation, is computed and compared to the one in absence of flow. A similar study, with subsonic flows is also presented. Different effects are observed in the low frequency region and around the acoustic coincidence.

\section{Funding Sources}

This project has received funding from the European Unions Horizon 2020 research and innovation programme under the Marie Sklodowska-Curie grant agreement No. 675441 (VIPER Project).

\section{Acknowledgments}

The authors would like to gratefully acknowledge Prof. E. Dowell, from Duke University, for the fruitful discussions. 


\section{References}

[1] Dowell, E., Aeroelasticity of Plates and Shells, Mechanics: Dynamical Systems, Springer Netherlands, 1974. URL https://books.google.fr/books?id=qRpof4bV-VoC

[2] Bisplinghoff, R., Aeroelasticity, Addison-Wesley series in mechanics, Addison-Wesley Pub. Co., 1955. URL https: //books.google. fr/books?id=uP9TAAAAMAA]

[3] E.H. Dowell, C. V., "Flutter of Low Aspect Ratio Plates," AIAA Journal, Vol. 8 (6), 1970, pp. 1162-1164. URL https: //arc.aiaa.org/doi/abs/10.2514/3.5858

[4] Miles, J., "On the Aerodynamic Instability of Thin Panels," Journal of the Aeronautical Sciences, Vol. 23 (8), 1956, pp. 771-791. URL https://arc.aiaa.org/doi/abs/10.2514/8.3653.

[5] Miles, J., "Supersonic Flutter of A Cylindrical Shell," Journal of the Aeronautical Sciences, Vol. 24, 1957, pp. 107-118. URL https://arc.aiaa.org/doi/abs/10.2514/8.3780

[6] Dugundji, J., Dowell, E., and Perkin, B., "Subsonic Flutter of Panels on Continuous Elastic Fundations,” AIAA Journal, Vol. 1 (5), 1963, pp. 1146-1154. URL/https://arc.aiaa.org/doi/abs/10.2514/3.1738

[7] Atkins, D. J., "The Effect of Uniform Flow on the Dynamics and Acoustics of Force-Excited Infinite Plates," No. AMTE (N)/TM82087 - Admiralty Marine Technology Establishment Teddington, 1982. URL https://apps.dtic.mil/docs/ citations/ADA124576

[8] Lucey, A. D., "The excitation of waves on a flexible panel in a uniform flow," Philosophical Transactions of the Royal Society of London. Series A: Mathematical, Physical and Engineering Sciences, Vol. 356, No. 1749, 1998, pp. $2999-3039$. doi:10.1098/rsta.1998.0306, URL https://royalsocietypublishing.org/doi/abs/10.1098/rsta.1998.0306

[9] Crighton, D. G., and Oswell, J. E., "Fluid loading with mean flow. I. Response of an elastic plate localized excitation," Philosophical Transactions of the Royal Society of London. Series A: Physical and Engineering Sciences, Vol. 335, No. 1639, 1991, pp. 557-592. URL/https://royalsocietypublishing.org/doi/abs/10.1098/rsta.1991.0060

[10] Sinha, B. K., Plona, T. J., Kostek, S., and Chang, S., "Axisymmetric wave propagation in fluid-loaded cylindrical shells. I: Theory," The Journal of the Acoustical Society of America, Vol. 92, No. 2, 1992, pp. 1132-1143. doi:10.1121/1.404040, URL https://doi.org/10.1121/1.404040

[11] Peake, N., "On the behaviour of a fluid-loaded cylindrical shell with mean flow," Journal of Fluid Mechanics, Vol. 338, 1997, p. 387-410. URL https://doi.org/10.1017/S0022112097005144

[12] Graham, W., "A Comparison of models for the wavenumber-frequency spectrum of turbolent boundary layer pressures," Journal of Sound and Vibration, Vol. 206(4), 1997, pp. 541-565. URL https://doi.org/10.1006/jsvi.1997.1114 
[13] Vitiello, P., Rosa, S. D., and Franco, F., "Convected field analysis of flat panels response to turbulent boundary layer induced excitation," Aerospace Science and Technology, Vol. 12, No. 1, 2008, pp. 91 - 104. doi:https://doi.org/10.1016/j.ast.2007.10.003, URL http://www.sciencedirect.com/science/article/pii/S1270963807001241

[14] Errico, F., Ichchou, M., De Rosa, S., Bareille, O., and Franco, F., "The modelling of the flow-induced vibrations of periodic flat and axial-symmetric structures with a wave-based method," Journal of Sound and Vibration, Vol. 424, 2018 , pp. 32-47. doi:/10.1016/j.jsv.2018.03.012.

[15] Errico, F., Ichchou, M., Franco, F., De Rosa, S., Bareille, O., and Droz, C., "Schemes for the sound transmission of flat, curved and axisymmetric structures excited by aerodynamic and acoustic sources," Journal of Sound and Vibration, Vol. 476, 2019, pp. 221-238. URL https://doi.org/10.1016/j.jsv.2019.05.041

[16] Brillouin, L., "Wave Propagation in Periodic Structures: Electric Filters and Crystal Lattices," 2nd edition Dover Publications, , No. Mineola, New York, 1953. URL https://doi.org/10.1016/S0031-8914(53)80099-6.

[17] Manconi, E., and Mace, B. R., "Wave characterization of cylindrical and curved panels using a finite element method," The Journal of the Acoustical Society of America, Vol. 125, No. 1, 2009, pp. 154-163. URL https://doi.org/10.1121/1.3021418.

[18] DOWELL, E., "Flutter of multibay panels at high supersonic speeds," AIAA Journal, Vol. 2, No. 10, 1964 , pp. $1805-1814$. doi:10.2514/3.2669, URL https://doi.org/10.2514/3.2669

[19] DOWELL, E. H., "On the flutter of multibay panels at low supersonic speeds." AIAA Journal, Vol. 5, No. 5, 1967, pp. 1032-1033. doi:10.2514/3.4125, URL https://doi.org/10.2514/3.4125

[20] Leppington, F., Broadbent, E., and Heron, K., “The acoustic radiation efficiency from rectangular plates,” Proc. R. Soc., Vol. 382, 1982, pp. 245-271. URL https://doi.org/10.1098/rspa.1982.0100

[21] Rodden, W., Farkas, E., Malcom, H., and Kliszewski, A., Aerodynamic Influence Coefficients from Supersonic Strip Theory: Analytical Development and Computational Procedure, Defense Technical Information Center, 1962. URL https: //books.google. fr/books?id=pBi5twAACAA]

[22] Bendiksen, O., and Seber, G., "Fluid-structure interactions with both structural and fluid nonlinearities," Journal of Sound and Vibration, Vol. 315, No. 3, 2008, pp. 664 - 684. doi:https://doi.org/10.1016/j.jsv.2008.03.034, URL http: //WWW.sciencedirect.com/science/article/pii/S0022460X0800271X eUROMECH colloquium 483, Geometrically non-linear vibrations of structures.

[23] DOWELL, E. H., "Nonlinear oscillations of a fluttering plate." AIAA Journal, Vol. 4, No. 7, 1966, pp. 1267-1275. doi: 10.2514/3.3658, URL https://doi.org/10.2514/3.3658

[24] FUNG, Y. C., “SOME RECENT CONTRIBUTIONS TO PANEL FLUTTER RESEARCH,” AIAA Journal, Vol. 1, No. 4, 1963, pp. 898-909. doi:10.2514/3.1661, URL https://doi.org/10.2514/3.1661 
[25] Bendiksen, O. O., "Review of unsteady transonic aerodynamics: Theory and applications," Progress in Aerospace Sciences, Vol. 47, No. 2, 2011, pp. 135 - 167. doi:https://doi.org/10.1016/j.paerosci.2010.07.001, URL http://wWw.sciencedirect. com/science/article/pii/S0376042110000473

[26] Bendiksen, O., and Davis, G., Nonlinear traveling wave flutter of panels in transonic flow, ???? doi:10.2514/6.1995-1486, URL https://arc.aiaa.org/doi/abs/10.2514/6.1995-1486

[27] De Rosa, S., Pezzullo, G., Lecce, L., and Marulo, F., "Structural acoustic calculations in the low frequency range," AIAA Journal of Aircraft, Vol. 31, No. 6, 1994, pp. 1387 - 1394. URL https://doi.org/10.2514/3.46663

[28] Sgard, F., Atalla, N., and Nicolas, J., "Coupled FEM-BEM approach for mean flow effects on vibro-acoustic behavior of planar structures," AIAA Journal, Vol. 32, No. 12, 1994, pp. 2351-2358. doi:https://doi.org/10.2514/3.12299, URL https://doi.org/10.2514/3.12299

[29] Maestrello, L., "Radiation from and panel response to a supersonic turbulent boundary layer," Journal of Sound and Vibration, Vol. 10, No. 2, 1969, pp. 261 - 295. doi:https://doi.org/10.1016/0022-460X(69)90200-4, URL http://www . sciencedirect com/science/article/pii/0022460X69902004

[30] Kariappa, O., Shah, C., and Somashekar, B., "Discrete element approach to flutter of skew panels with in-plane forces under yawed supersonic flow," AIAA Journal, Vol. 8 (11), 1970, pp. 2017-2022. URL https://arc.aiaa.org/doi/abs/10. $2514 / 3.6040$

[31] Krumhaar, H., "The accuracy of linear piston theory when applied to cylindrical shells," AIAA Journal, Vol. 1 (6), 1963, pp. 1448-1449. URL https://arc.aiaa.org/doi/abs/10.2514/3.1832

[32] Olson, M. D., Supersonic Flutter of Circular Cylindrical Shells, PhD Thesis, California Institute of Technology, 1966. URL http://resolver.caltech.edu/CaltechETD:etd-12022005-075701

[33] Leissa, A., "Vibration of Plates," Scientific and Technical Information Division, National Aeronautics and Space Administration, Vol. 160, 1969. URL https://ntrs.nasa.gov/search.jsp?R=19700009156

[34] Leissa, A., "Vibration of Shells," Scientific and Technical Information Division, National Aeronautics and Space Administration, Vol. SP-288, 1973. URL https://ntrs.nasa.gov/search.jsp?R=19730018197

[35] Cockburn, J., and Robertson, J., "Vibration response of spacecraft shrouds to in-flight fluctuating pressures," Journal of Sound and Vibration, Vol. 33, No. 4, 1974, pp. 399-425. URL https://www.sciencedirect.com/science/article/pii/ S0022460X74802269.

[36] Corcos, G., "Resolution of pressure in turbulence," Journal of the Acoustical Society of America, Vol. 35, 1963 , pp. $192-199$. URL https://asa.scitation.org/doi/10.1121/1.1918431 